\title{
Endoscopic Assisted Microscopic Fenestration of Inner Membrane in Cases of Chronic Subdural Hematoma
}

\author{
Mohamed Gaber Abdel Tawab ${ }^{1}$, Mohamed Reda ${ }^{2}$, Ahmed El-Fiki ${ }^{2}$ \\ ${ }^{1}$ Department of Neurosurgery, Fayoum University, Fayoum, Egypt \\ ${ }^{2}$ Department of Neurosurgery, Cairo University, Giza, Egypt \\ Email:gaber_28@yahoo.com
}

How to cite this paper: Tawab, M.G.A., Reda, M. and El-Fiki, A. (2019) Endoscopic Assisted Microscopic Fenestration of Inner Membrane in Cases of Chronic Subdural Hematoma. Open Journal of Modern Neurosurgery, 9, 172-183.

https://doi.org/10.4236/ojmn.2019.92017

Received: March 15, 2019

Accepted: April 25, 2019

Published: April 28, 2019

Copyright $\odot 2019$ by author(s) and Scientific Research Publishing Inc. This work is licensed under the Creative Commons Attribution International License (CC BY 4.0).

http://creativecommons.org/licenses/by/4.0/ (c) (i) Open Access

\begin{abstract}
Introduction: Chronic subdural hematoma (CSDH) is one of the most common types of intracranial hemorrhages and carries a significant morbidity; there is no clear optimal treatment. Recurrence, pneumocephaly and seizures are common complications. We will evaluate outcome after inner membrane fenestration in comparison to burr-hole evacuation only in cases of CSDH. Patients and Methods: Our work was conducted on 20 patients with CSDH from February 2017 to July 2017. Endoscopic-assisted microscopic fenestration technique was used to do inner membrane fenestration in ten patients and the other 10 patients operated upon by traditional burr-hole evacuation. Regular follow up was done up to one month. Results: Clinical outcome was nearly the same in the two groups. Recurrence rate was only noted in the non-fenestration group (20\%). Midline shift was better in the membrane fenestration group after one month and we experienced no intraoperative surgical complications related to the fenestration technique. Postoperative complications, such as Pneumocephalus and seizures, were seen in both study groups. Conclusion: Endoscopic assisted microscopic technique decreases surgical risks to do inner membrane fenestration although there is no major difference between doing fenestration or not in clinical outcome, but it may decrease recurrence, but larger studies are needed.
\end{abstract}

\section{Keywords}

Endoscopic Assisted Microscopic, Chronic Subdural Hematoma (CSDH), Membranectomy, Fenestration

\section{Introduction}

The incidence of chronic subdural hematoma (CSDH) in the general population 
has been rising. This is, in part, due to an increase in the elderly population and an increase in the use of oral antiplatelet and anticoagulation agents in this group of patients [1] [2]. Patients often live with chronic, subacute, or mixed acute on top of CSDH in the community and do not present to medical attention until they become symptomatic [3].

The most common theory to explain CSDH is that minor inertial brain injury causes movement of the brain within the skull and tears bridging veins as they traverse cell layer of the dural border [4].

Gardner [5] suggested that a difference in osmolarity between cerebrospinal fluid (CSF) and CSDH fluid cause its enlargement by imbibing CSF. Subsequent theories invoked oncotic pressure gradients between intravascular plasma and CSDH fluid. The osmotic/oncotic pressure gradient theory is not supported by studies showing there is no difference in osmolarity or oncotic pressure among CSDH fluid, plasma, and CSF [6].

Although recurrent hemorrhage is probably the most important process that leads to CSDH enlargement, it is likely that exudation and transudation contribute in part to the pathogenesis of CSDH.

Lee et al. [7] suggested that CSDH may develop following hemorrhage into a subdural hygroma. The development of a hygroma, much like a hematoma, induces the formation of neo-membranes accompanied by neovascularization, and these fragile vessels promote repeated microhemorrhage and eventual development of CSDH [8].

Many studies have explored the various treatment modalities for CSDH. These treatment options have included craniotomies, burr-hole evacuation (BHE), and twist drill craniotomies (TDC).

Neurosurgeons have been focusing on less invasive techniques in many of these patients to minimize complications. Various studies have compared the outcomes from less invasive TDC with craniotomies and BHE. These studies have reported similar radiographic and clinical outcomes among the groups treated with different techniques.

Endoscopic-assisted evacuation of CSDH is a well-established technique, although not commonly used. It is combined of burr-hole evacuation of CSDH with inspection of the subdural space with the use of the endoscope [9]. Endoscopic assisted microscopic techniques have not been previously discussed in literature.

We aim to evaluate the effect of partial excision of the inner membrane of chronic subdural hematoma with BHE by endoscopic assisted microscopic technique compared to evacuation without membranectomy in terms of clinical and radiological outcome in the first month postoperatively.

\section{Patients and Methods}

This study was prospectively conducted on 20 patients with chronic subdural hematomas operated upon in the period from February 2017 to July 2017 in 
Neurosurgery Department at Fayoum University. We included all the patients with CSDH (proved by CT and or MRI) operated for the first time and Patients of both genders and age groups from 5th to the 9th decades with different etiologies.

Exclusion criteria include patients with recurrent CSDH after previous operation, patients with calcified hematomas and asymptomatic patients with thin film CSDH.

All patients were subjected to thorough history taking and clinical examination with careful evaluation of pre-existing chronic illnesses especially those associated with coagulopathy or thrombopathy or those on regular medications especially antiepileptic drugs, anticoagulants and antiplatelet agents. Complete laboratory work-up was routinely carried out. CT brain was done for all cases and MRI of the brain was performed for cases isodense hematomas.

Patients were classified into two groups. Fenestration group: Ten patients were operated upon via burr-hole evacuation with inner membranectomy then drainage into a closed drainage system. Non-fenestration group: Ten patients were operated upon via BHE then drainage into a closed drainage system without inner membranectomy.

\section{Surgical Technique}

All the patients were operated upon under general anesthesia in the supine position, the head was turned to the contralateral side of the hematoma at a 45-degree angle.

In fenestration group: Two burr-holes were made and enlarged with a kerrison rongeur $(15 \mathrm{~mm})$. The dura was cauterized using a bipolar cautery, then incised in a cruciate manner and opened. Edges of the dura were coagulated on the edges of the burr-hole. The outer membrane of the hematoma was opened using a number 15 scalpel. Subdural cavity was irrigated using normal saline until the color of the fluid become clear.

The surgical microscope was used to visualize the cortex through the anterior burr-hole. Surgical endoscope was brought through the posterior burr-hole and manipulated by the help of the assistant surgeon. The inner membrane was lifted by a forceps and 3 to $5 \mathrm{~cm}$ were opened initially by microscissors then hydrodissection via insulin syringe, so it was easily torn out (Figure 1).

The surgical microscope allowed better illumination and magnification through the anterior burr-hole and facilitates accurate site of the inner membrane opening (area devoid of cortical vessels), different endoscopic angles cleared out residual hematoma not amenable to be seen with the surgical microscope.

After subdural space (SDS) irrigation was completed, the tip of nelaton catheter was inserted through the posterior parietal burr-hole and the proximal tip catheter with $5-7 \mathrm{~cm}$ left in SDS. SDS was filled with normal saline and connected to a closed drainage system. The drain was externalized from the posterior 


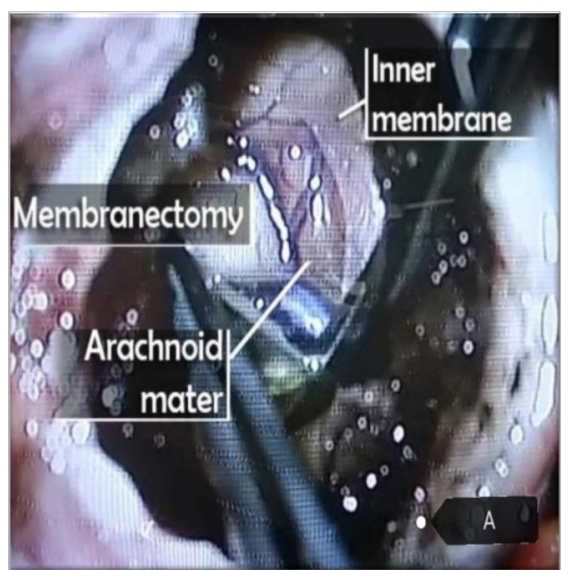

(a)

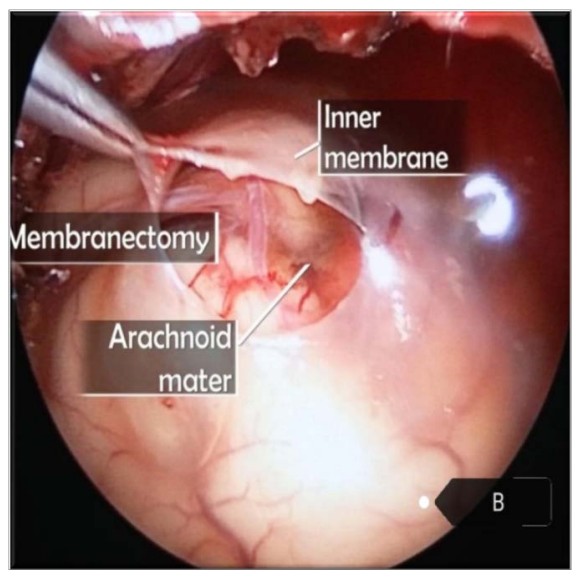

(b)

Figure 1. Inner membranectomy technique in the fenestration group. (a) An intraoperative microscopic image showing the inner membrane of the hematoma held by a forceps while membranectomy is being done using a microscissor; (b) An intraoperative endoscopic image showing an opening made in the intact inner membrane which is held by a forceps. The underlying arachnoid mater can be seen in both views.

parietal burr-hole and fixed to the scalp carefully. The drain was removed after 48 - 72 hours.

In Non-fenestration group, the steps are the same, except for the inner membranectomy part and using the endoscopic assisted microscopic technique.

Antibiotics and prophylactic antiepileptic drugs were routinely given to all the patients postoperatively.

Full neurological examination was performed to all patients to detect improvement or deterioration of the neurological condition. Post-operative CT brain was done on the $1^{\text {st }}$ and $3^{\text {rd }}$ postoperative days to assess expansion of the brain, decrease in the subdural space thickness, improvement of midline shift, occurrence of pneumocephalus and reperfusion injuries. Regular follow up till one month in outpatient clinic with CT brain to detect any recollection of the hematoma.

\section{Statistical Analysis}

Data were collected and coded to facilitate data manipulation and double entered Microsoft Access and data analysis was performed using SPSS software version 18 in windows 7. Simple descriptive analysis in the form of numbers and percentages for qualitative data, and arithmetic means as central tendency measurement, standard deviations as measure of dispersion for quantitative parametric data, and inferential statistic test; For quantitative parametric data Independent $t$-Test used to compare measures of two independent groups of quantitative data and paired t-test in comparing two dependent quantitative data. For qualitative data Chi-square test to compare two of more than two qualitative groups and Mc-Nemar test for paired dependent qualitative data. The p-value $\leq 0.05$ was considered the cut-off value for significance. 


\section{Sampling}

The sample size was calculated according to Epi Info 2000. A sample size was selected using a special formula based on the prevalence of disease at a confidence interval of $95 \%$ and precision of $(2 \%)$. The sample increased by $10 \%$ to overcome problems related to non-responses and missing data. The power of study was $80 \%$.

\section{Ethical Approval}

The study was approved by the scientific research ethical committee at our University hospital after ensuring the ethical standards and scientific merit of research involving our patients and was matched with the 1964 Helsinki Declaration and its later amendments.

\section{Consenting}

Informed Consents were obtained from the patients and/or their legal representatives after being informed of the details of the surgery, the probable outcome, the complications, and the possibility of recurrence and re-operation.

\section{Results}

On comparing the demographic characteristics of the patients in study groups, both were nearly the same regarding sex distribution and age range with mean age in 6 th decade (Table 1 ).

There was no statistically significant difference with p-value $>0.05$ between study groups regarding age and sex distribution which indicated proper matching between the groups.

History of mild head trauma was found in $60 \%$ - $70 \%$ of patients from both groups. Six patients $(30 \%)$ included in our study needed preoperative correction of coagulopathy or thrombopathy. Four of them were in fenestration group and 2 were in non-fenestration group.

Glasgow coma scale (GCS) was 12 or more in 7 patients in both groups. Epileptic seizures were reported in only 1 patient in Non-fenestration group, none in fenestration group. Motor power in all patients of fenestration group was

Table 1. Comparison between demographic characters in different study groups. ${ }^{\star}$ Sig: significance ${ }^{\star}$ NS: non significant.

\begin{tabular}{|c|c|c|c|c|c|c|}
\hline \multirow{2}{*}{ variables } & \multicolumn{4}{|c|}{ Technique } & \multirow{2}{*}{$\mathrm{p}$-value } & \multirow{2}{*}{ Sig. } \\
\hline & Fen & group & Non-f & n group & & \\
\hline $\begin{array}{l}\text { Age (mean in } \\
\text { years) }\end{array}$ & \multicolumn{2}{|c|}{70.5} & \multicolumn{2}{|c|}{67.2} & $>0.05$ & NS \\
\hline \multicolumn{7}{|c|}{ Sex } \\
\hline Male & 7 & $70 \%$ & 8 & $80 \%$ & \multirow[b]{2}{*}{$>0.05$} & \multirow[b]{2}{*}{ NS } \\
\hline Female & 3 & $30 \%$ & 2 & $20 \%$ & & \\
\hline
\end{tabular}


grade IV or below while in Non-fenestration group, two patients were full motor power.

According to the Markwalder grading system, in fenestration group, 5 patients (50\%) were grade 2 and 5 patients (50\%) were grade 3 . In non-fenestration group, 1 patient (10\%) was grade 1,3 patients (30\%) were grade 2 and 6 patients (60\%) were grade 3.

There were no grade 0 patients in this study because all the patients in the sample group were symptomatic, requiring surgical drainage. The improvement of patients inside each group was statistically significant $(\mathrm{p}<0.05)$ but there was no significant difference between the 2 groups (Table 2).

Unilateral hematomas were encountered in $80 \%$ of cases, while 2 patients (20\%) presented with bilateral hematoma.

Average preoperative midline shift in fenestration group was $11 \pm 4.3 \mathrm{~mm}$ (range: 0 - $16 \mathrm{~mm}$ ), while in Non-fenestration group was $10.5 \pm 4.11 \mathrm{~mm}$ (range: $0-14 \mathrm{~mm}$ ). The difference in midline shift between the 2 groups after one month proved to be statistically significant (Table 3 ).

All patients showed improvement of motor power postoperatively reaching full motor power on follow up visit after one month.

None of Pneumocephalus was seen in postoperative CT of eleven patients (55\%) of both study groups. None of these patients needed tapping or reoperation as pneumocephalus has been resolving spontaneously. In fenestration

Table 2. Comparison of pre- and post-operative evaluation in different study groups according to Markwalder's grading system for CSDH.

\begin{tabular}{|c|c|c|c|c|c|c|}
\hline \multirow{3}{*}{$\begin{array}{c}\text { Markwalder } \\
\text { grade }\end{array}$} & \multicolumn{4}{|c|}{ Technique } & \multirow{3}{*}{$\mathrm{p}$-value } & \multirow{3}{*}{ Sig. } \\
\hline & \multicolumn{2}{|c|}{ Fenestration group } & \multicolumn{2}{|c|}{ Non-fenestration group } & & \\
\hline & No. & $\%$ & No. & $\%$ & & \\
\hline \multicolumn{7}{|c|}{ Before operation } \\
\hline Grade 0 & 0 & $0 \%$ & 0 & $0 \%$ & $>0.05$ & NS \\
\hline Grade 1 & 0 & $0 \%$ & 1 & $10 \%$ & $>0.05$ & NS \\
\hline Grade 2 & 5 & $50 \%$ & 3 & $3 \%$ & $>0.05$ & NS \\
\hline Good & 5 & $50 \%$ & 4 & $40 \%$ & $>0.05$ & NS \\
\hline Grade 3 & 5 & $50 \%$ & 6 & $50 \%$ & $>0.05$ & NS \\
\hline Grade 4 & 0 & $0 \%$ & 0 & $0 \%$ & $>0.05$ & NS \\
\hline $\mathrm{Bad}$ & 5 & $50 \%$ & 6 & $60 \%$ & $>0.05$ & NS \\
\hline \multicolumn{7}{|c|}{ After operation } \\
\hline Grade 0 & 2 & $20 \%$ & 1 & $10 \%$ & $>0.05$ & NS \\
\hline Grade 1 & 1 & $10 \%$ & 2 & $20 \%$ & $>0.05$ & NS \\
\hline Grade 2 & 7 & $70 \%$ & 7 & $70 \%$ & $>0.05$ & NS \\
\hline Good & 10 & $100 \%$ & 10 & $100 \%$ & $>0.05$ & NS \\
\hline Grade 3 & 0 & $0 \%$ & 0 & $0 \%$ & $>0.05$ & NS \\
\hline Grade 4 & 0 & $0 \%$ & 0 & $0 \%$ & $>0.05$ & NS \\
\hline $\mathrm{Bad}$ & 0 & $0 \%$ & 0 & $0 \%$ & $>0.05$ & NS \\
\hline
\end{tabular}


Table 3. Comparison between the average pre- and post-operative midline shift in different study groups.

\begin{tabular}{|c|c|c|c|c|c|c|}
\hline \multirow{3}{*}{$\begin{array}{l}\text { Average } \\
\text { Midline shift } \\
\quad(\mathrm{mm})\end{array}$} & \multicolumn{4}{|c|}{ Technique } & \multirow{3}{*}{$\mathrm{p}$-value } & \multirow{3}{*}{ Sig. } \\
\hline & \multicolumn{2}{|c|}{ Fenestration group } & \multicolumn{2}{|c|}{ Non-fenestration group } & & \\
\hline & Mean & $\mathrm{SD}$ & Mean & $\mathrm{SD}$ & & \\
\hline Preoperative & 11 & 4.3 & 10.5 & 4.11 & $>0.05$ & NS \\
\hline $\begin{array}{c}1^{\text {st }} \& 3^{\text {rd }} \text { postop. } \\
\text { Days }\end{array}$ & 4 & 2.1 & 5 & 3.05 & $>0.05$ & NS \\
\hline After one month & 0.2 & 0.42 & 0.8 & 0.78 & 0.05 & $S$ \\
\hline
\end{tabular}

${ }^{*}$ Sig: significance, ${ }^{*}$ S: significant.

group, three patients (30\%) had postoperative attacks of seizures. In non-fenestration group, seizures occurred in two cases (20\%).

None of the patients in fenestration group had recurrence of the hematoma but 2 patients (20\%) in non-fenestration group had recurrence. One of them was clinically intact with no midline shift seen in the $\mathrm{CT}$ and was managed conservatively till spontaneous absorption. The other patient presented with recurrent hemiparesis and was re-operated by craniotomy, evacuation of clotted hematoma and excision of membrane and septations. In clinical practice, this difference in recurrence rate can be considered significant, but after statistical analysis, it was insignificant due to the relatively small sample size.

Average hospital stay in both groups of our study was $4.1 \pm 1.6$ days (range: 3 - 8 days). We had no cases of mortality in both groups.

\section{Discussion}

Chronic subdural hematoma is one of the most common neurosurgical conditions. It is frequently encountered in elderly people, usually after minor head trauma, and in patients on long-term hemodialysis and long-term anticoagulation. Bilateral chronic subdural hematomas tend to occur more frequently in patients on anticoagulant or antiplatelet therapy .

Most cases of CSDH need surgical intervention. Common surgical procedures for CSDH include TDC, BHE or craniotomy and some less common adjunctive techniques.

Both the systematic review by Weigel et al. [10] and a decision analysis model based on the data reported in the literature by Lega et al. [3] have identified BHE as the most efficient choice to treat a primary and uncomplicated CSDH because it balances a low recurrence rate against a better morbidity and mortality profile compared to craniotomy and TDC.

Since the 1990s, evidence has been emerging that the use of drains with BHE is associated with lower recurrence rates [11] [12] [13] [14] [15]. Several groups have attempted to address the role of irrigation in the treatment of CSDH and have demonstrated a trend toward reduced recurrence with the use of intraoperative irrigation [16] [17] [18]. Only Kuroki et al. [19] found more than 6 times 
higher recurrence $(\mathrm{p}=0.49)$ in cases with irrigation ( 5 of 45 patients, $11.1 \%$ ) than without irrigation ( 1 of 55 patients, $1.8 \%$ ).

Regarding the BHE technique, most surgeons use two burr-holes mainly because this allows better washout of the subdural cavity. Taussky et al. [20] observed a higher recurrence rate if one rather than two burr-holes was used. Lega et al. [3] revealed BHE results in significantly better outcomes than TDC or craniotomy.

In 1988, Karakhan et al. published the use of the surgical endoscope for evacuation of CSDH [21]. Since then, several other authors have introduced slight modifications of the technique [22]. Recently, several reports of endoscopic evacuation of subacute and acute subdural hematomas have appeared in the literature [23]. When reviewing the literature, we have found no studies discussing compining the use of endoscope with the microscope for evacuation of CSDH.

The major risk factors influencing recurrence include hematoma density, presence of postoperative pneumocephalus, alcoholism, history of coagulopathy or thrombopathy and intake of anticoagulant or antiplatelet medications. Poor brain re-expansion ability has been suggested for development of recurrence [24].

Putnam and Cushing propose craniotomy and excision of the outer and inner membranes to prevent recurrence [25]. Manipulating the inner membrane found to result in brain re-expansion easily, and consequently, results in less incidence of pneumocephalus and recurrence despite it is considered risky or invasive.

During the procedure, cortical vessels may be injured, and intracerebral or acute subdural hemorrhage may occur, a complication that was avoided by using surgical microscope to open through and area $3-5 \mathrm{~mm}$ with no underling cortical vessels.

Inner membranectomy with the endoscopic assisted microscopic technique, is considered a novel idea. After reviewing the literature, we found that this subject was only discussed once in a retrospective study done in 2014 by Kayaci et al. using a microscopic technique [26].

Local brain herniation through inner membranectomy was reported before, But this incident did not happen to any of our patient [26].

We adopted a newer technique when we used the surgical microscope through the frontal burr-hole while introducing the surgical endoscope through the parietal buur-hole in the same time. This technique minimizes the surgical risks of cortical injury while opening the inner membrane.

In the study done by Kayaci et al., only $4 \%$ of the patients were on anticoagulants and needed correction [26]. Lee et al. found that $25 \%$ of their patients had impaired coagulation profile [8]. In our study, $30 \%$ of the patients needed preoperative correction of coagulopathy or thrombopathy. This may be due to the prevalence of liver diseases in Egypt which in turn negatively affects the process 
of coagulation and platelet count or function.

Although peumocephalus percent inpost-operative CT was higher in our study (55\%) than what was found by Kayaci et al. [26] (almost 40\% of their patients). but our cases did not require any tapping or re-operation like what they did for $16 \%$ of their patients.

Unlike what was reported by Kayaci et al. and Wang et al. [26] [27] about higher incidence of postoperative seizures in non membranectomy patients, in our cases patients who developed seizures was in fenestration group, but all were controlled by antiepileptics.

Our patients stayed in the hospital for shorter period ( $4.1 \pm 1.6$ days) than what was proved by Kayaci et al. [26] (hospital stay was $8.8 \pm 0.2$ ) and this may be explained by decreasing the surgical complications by using the microscopic assisted endoscopic technique with better visualization.and subsequently decreasing hospital stay.

Our recurrence cases were reported innon-fenestration group (20\%) and that was similar to recurrence cases of Kayaci et al. study (8.3\%) but none of fenestration group patients develop recurrence in both studies. Unlike Lee et al. who had recurrence in his study reaching up to $23 \%$ in fenestration group, but these cases were with either craniotomy or craniectomy [8].

Kim et al. documented recurrence in $8.9 \%$ of the patients in group I (BHE without membranectomy group), $50 \%$ in group II (the small craniotomy with membranectomy group) and $9.5 \%$ of the patients in group III (the large craniotomy with membranectomy group) [28].

When comparing the time of surgery in both procedures, fenestration technique was expected to have longer operating time. The traditional burr-hole evacuation took an average surgery time of 30 - 40 minutes. In the first few cases, the fenestration step used to consume more operating time as we were not familiar with the technique yet, but later it used to take extra $10-15$ minutes with a total operating time of 40 - 55 minutes. This extra time did not make a statistically significant difference between the study groups. We had no detectable blood loss in both study groups.

In our study, we had no cases of mortality. Similarly, Zakaria et al. had no incidence of mortality in their series [18]. On the other hand, Oktay et al. reported mortality in $4 \%$ of the cases in their study [29].

Our study limitations include small number of patients and short follow up period which can be adjusted in future studies.

\section{Conclusion}

Burr-hole evacuation with or without inner membranectomy provides excellent outcome in the treatment of chronic subdural hematoma. Endoscopic-assisted microscopic technique decreases surgical risks to do inner membrane fenestration although there is no major difference between doing fenestration or not in clinical outcome, but it may decrease recurrence, but larger studies are needed. 


\section{Acknowledgements}

I would like to thank Ahmed Owis (MSc Neurosurgery, Fayoum University) for the kind support with collecting data, helping in the surgical field and kindness to our patients.

\section{Conflicts of Interest}

This study has not been submitted to, nor is under review at, another journal or other publishing venue. The authors have no affiliation with any organization with a direct or indirect financial interest in the subject matter discussed in the study.

\section{References}

[1] Kolias, A.G., Chari, A., Santarius, T. and Hutchinson P.J. (2014) Chronic Subdural Haematoma: Modern Management and Emerging Therapies. Nature Reviews Neurology, 10, 570-578. https://doi.org/10.1038/nrneurol.2014.163

[2] Rust, T., Kiemer, N. and Erasmus, A. (2006) Chronic Subdural Haematomas and Anticoagulation or Antithrombotic Therapy. Journal of Clinical Neuroscience, 13, 823-827. https://doi.org/10.1016/j.jocn.2004.12.013

[3] Lega, B.C., Danish, S.F., Malhotra, N.R., Sonnad, S.S. and Stein, S.C. (2010) Choosing the Best Operation for Chronic Subdural Hematoma: A Decision Analysis. Journal of Neurosurgery, 113, 615-621. https://doi.org/10.3171/2009.9.jns08825

[4] Yamashima, T. and Friede, R.L. (1984) Why Do Bridging Veins Rupture into the Virtual Subdural Space? Journal of Neurology, Neurosurgery and Psychiatry, 47, 121-127. https://doi.org/10.1136/jnnp.47.2.121

[5] Gardner, W.J. (1932) Traumatic Subdural Hematoma with Particular Reference to the Latent Interval. Archives of Neurology and Psychiatry, 27, 847-858. https://doi.org/10.1001/archneurpsyc.1932.02230160088009

[6] Weir, B. (1980) Oncotic Pressure of Subdural Fluids. Journal of Neurosurgery, 53, 512-515. https://doi.org/10.3171/jns.1980.53.4.0512

[7] Lee, K.S., Bae, W.K., Park, Y.T. and Yun, I.G. (1994) The Pathogenesis and Fate of Traumatic Subdural Hygroma. British Journal of Neurosurgery, 8, 551-558. https://doi.org/10.3109/02688699409002947

[8] Lee, J.Y., Ebel, H., Ernestus, R.I. and Klug, N. (2004) Various Surgical Treatments of Chronic Subdural Hematoma and Outcome in 172 Patients: Is Membranectomy Necessary? Surgical Neurology, 61, 523-527. https://doi.org/10.1016/j.surneu.2003.10.026

[9] Masopust, V., Netuka, D. and Häckel, M. (2003) Chronic Subdural Haematoma Treatment with a Rigid Endoscope. Minimally Invasive Neurosurgery, 46, 374-379. https://doi.org/10.1055/s-2003-812507

[10] Weigel, R., Schmiedek, P. and Krauss, J.K. (2003) Outcome of Contemporary Surgery for Chronic Subdural Haematoma: Evidence Based Review. Journal of Neurology, Neurosurgery and Psychiatry, 74, 937-943.

https://doi.org/10.1136/jnnp.74.7.937

[11] Lind, C.R.P., Lind, C.J. and Mee, E.W. (2003) Reduction in the Number of Repeated Operations for the Treatment of Subacute and Chronic Subdural Hematomas by Placement of Subdural Drains. Journal of Neurosurgery, 99, 44-46. 
https://doi.org/10.3171/ins.2003.99.1.0044

[12] Wakai, S., Hashimoto, K., Watanabe, N., et al. (1990) Efficacy of Closed System Drainage in Treating Chronic Subdural Hematoma: A Prospective Comparative Study. Neurosurgery, 26, 771-773. https://doi.org/10.1227/00006123-199005000-00006

[13] Okada, Y., Akai, T., Okamoto, K., et al. (2002) A Comparative Study of the Treatment of Chronic Subdural Hematoma-Burr Hole Drainage versus Burr Hole Irrigation. Surgical Neurology, 57, 405-409. https://doi.org/10.1016/s0090-3019(02)00720-6

[14] Ramachandran, R. and Hegde, T. (2007) Chronic Subdural Hematomas-Causes of Morbidity and Mortality. Surgical Neurology, 67, 367-372.

https://doi.org/10.1016/j.surneu.2006.07.022

[15] Asano, Y., Hasuo, M., Takahashi, I. and Shimosawa S. (1992) Recurrent Cases of Chronic Subdural Hematoma-Its Clinical Review and Serial CT Findings. No to Shinkei, 44, 827-831.

[16] Matsumoto, K., Akagi, K., Abekura, M., et al. (1999) Recurrence Factors for Chronic Subdural Hematomas after Burr-Hole Craniostomy and Closed System Drainage. Neurological Research, 21, 277-280. https://doi.org/10.1080/01616412.1999.11740931

[17] Gurelik, M., Aslan, A., Gurelik, B., et al. (2007) A Safe and Effective Method for Treatment of Chronic Subdural Haematoma. Canadian Journal of Neurological Sciences, 34, 84-87. https://doi.org/10.1017/s0317167100005849

[18] Zakaraia, A.M., Adnan, J.S., Haspani, M.S., et al. (2008) Outcome of 2 Different Types of Operative Techniques Practiced for Chronic Subdural Hematoma in Malaysia: An Analysis. Surgical Neurology, 69, 608-615. https://doi.org/10.1016/j.surneu.2007.01.054

[19] Kuroki, T., Katsume, M., Harada, N., et al. (2001) Strict Closed-System Drainage for Treating Chronic Subdural Haematoma. Acta Neurochirurgica (Wien), 143, 1041-1044. https://doi.org/10.1007/s007010170010

[20] Taussky, P., Fandino, J. and Landolt, H. (2008) Number of Burr Holes as Independent Predictor of Postoperative Recurrence in Chronic Subdural Haematoma. British Journal of Neurosurgery, 22, 279-282. https://doi.org/10.1080/02688690701818885

[21] Karakhan, V.B. (1988) Experience Using Intracranial Endoscopy in Neurologic Traumatology. Vestnik Khirurgii Imeni I. I. Grekova, 140, 102-108.

[22] Çokluk, C., Kuruoğlu, E., Marangoz, A.H. and Aydin, K. (2014) The Neuroendoscopic Assisted Microsurgical Evacuation of Chronic Subdural Hematomas. Journal of Clinical and Analytical Medicine, 5, 287-290. https://doi.org/10.4328/jcam.1353

[23] Codd, P.J., Venteicher, A.S., Agarwalla, P.K., Kahle, K.T. and Jho, D.H. (2013) Endoscopic Burr Hole Evacuation of an Acute Subdural Hematoma. Journal of Clinical Neuroscience, 20, 1751-1753. https://doi.org/10.4328/jcam.1353

[24] Kurabe, S. (2010) Efficacy and Safety of Postoperative Early Mobilization for Chronic Subdural Hematoma in Elderly Patients. Acta Neurochirurgica (Wien), 152, 1171-1174. https://doi.org/10.1007/s00701-010-0627-4

[25] Garg, R.K. (2017) Chronic Subdural Hematoma. MedLink Neurology.

[26] Kayaci, S., Kanat, A., Koksal, V. and Ozdemir, B. (2014) Effect of Inner Membrane Tearing in the Treatment of Adult Chronic Subdural Hematoma: A Comparative Study. Neurologia Medico-Chirurgica, 54, 363-373. 
[27] Wang, K., Chen, D., Cao, X. and Gao, L. (2017) A Prospective Comparative Study of Twist Drill Craniostomy versus Burr Hole Craniostomy in Patients with Chronic Subdural Hematoma. Turkish Neurosurgery, 27, 60-65.

https://doi.org/10.5137/1019-5149.jtn.14519-15.1

[28] Kim, J.H., Kang, D.S., Kim, J.H., Kong, M.H. and Song, K.Y. (2011) Chronic Subdural Hematoma Treated by Small or Large Craniotomy with Membranectomy as the Initial Treatment. Journal of Korean Neurosurgical Society, 50, 103-108. https://doi.org/10.3340/jkns.2011.50.2.103

[29] Oktay, K., Olguner, S.K., Saraç, M.E., et al. (2016) Treatment of Chronic Subdural Hematoma: 5-Year Clinical Experience. Gaziantep Medical Journal, 22, 118-123. 